\title{
A generic method for the assessment of smart walkers
}

\author{
Pierre Rumeau $^{1}$, Nadine Vigouroux ${ }^{2}$, Ludovic Saint-Bauzel ${ }^{3}$ and Viviane Pasqui ${ }^{3 *}$ \\ ${ }^{1}$ Laboratoire de Gérontechnologie/ Gérontopôle / UMR 1027 Inserm-Université de \\ Toulouse, Hôpital La Grave, Toulouse, France \\ ${ }^{2}$ Université Paul Sabatier, Toulouse, France \\ ${ }^{3}$ ISIR, Sorbonne Universités-UPMC, Paris, France \\ *Corresponding author (pasqui@isir.upmc.fr)
}

\begin{abstract}
Purpose This paper presents a generic method to assess the usability of smart-walkers. With an increasing number of assistive robots available, it is important to make sure they work before making a choice. Mechatronic systems propose more functions that are of interest to the ageing frail or handicapped population than conventional walking frames. For example: helping the user to get up or to sit down, avoiding obstacles, finding directions, driving, etc. These systems are all research prototypes, except PAM-AID which was marketed under GUIDO ${ }^{\mathrm{TM}}$. Most of them were tested in order to validate their features either with healthy young subjects, or with elderly people. However, only PAMAID was tried for its usability. In that study the PAM-AID was compared to the AMD (Assistive Mobility Device developed by Atlanta VAMC which is totally passive and without sensors) and the user's usual walking aid. The test used in the evaluation protocol is a $36.6 \mathrm{~m}$ course, without obstacles, with each of the three different technical devices. The time to complete the test and a questionnaire were included in the analysis. Our reports suggest that a generic method is needed to evaluate smartwalkers whatever their robotics features. Method The usability of smartwalkers was assessed with a normalized test used in geriatric medicine to diagnose frailty and walking difficulties: the 4-meter walking test. If the volunteer failed the complete the 4 meters, the protocol specified the test would stop at that stage. Failure of the 4-meter test was defined as taking over ten times more time to complete the test with a device than it would take volunteer walking usually(i.e. without aid or a different aid such as a cane). During the test, the volunteer was first trained to complete the test, then to complete it with first the regular walking frame and then again with the robuWalker. Training lasted as long as the volunteer wanted to feel sufficiently confident. Each test was run immediately after training with a new device (usual way of walking; walking frame; robuWalker). The method adds to the time of completion a gait analysis including the feet motion extracted from videos. Results \& Discussion In a previous article we analyzed the completion time. However time is hardly sufficient to describe the required speed of an active smart walker, extra information such as trajectory, instant speed, distance to the marking, duration of double limb, etc. are also required. We applied to the $4 \mathrm{~m}$-test a gait analysis including feet motion analysis from the videos of our two groups of four healthy elderly volunteers and four impaired volunteers (with both motor and cognitive impairments) and three test conditions.
\end{abstract}

Keywords: elderly people, walking aids, robots, Geriatric assessment, method

\section{INTRODUCTION}

The increasing number of elderly people in our society, also ageing is most commonly successful, is causing an increase in the need for useful assistive devices. An effective walking aid (or walker) has to answer several criteria: be usable, efficient, stabilizing and of low energy cost to the user. These criteria are strongly linked. A pathological walk will be more expensive in terms of energy and a fall will have a greater risk to occur. Regular walking frames are rudimentary, they don't provide obstacle avoidance, can't help to stand securely from seated. The ideal device should supply a mechanical support and should be able to adapt itself to the environment and to the incapacities of the user. A robotic device is in practice most likely to be able to answer these specifications. Previous works have proposed smart-walkers addressing these needs.
Mechatronic systems allow additional functions such as:

- helping the user to get up or to sit down,

- to avoid an obstacle,

- to help in orientation,

- driving, etc...

The existing systems can be classified according to their features. A motorized smart-walker can work on two modes: passive or active. When it is passive; it is following the movements of the user, in this case the human is the master over the system. When it is active; it can move by itself and override, to some extent, the intentions of the user, in particular to avoid an obstacle. Both modes (passive or active) can use the same mechatronic system depending on the choice of various control modes on the motorized wheels. However, some systems are only passive ${ }^{1}$, or with active brakes only ${ }^{2}$ or active in direction only ${ }^{3,4}$. Most of the smart-walkers 
have motorized wheels, allowing an active assistance in weight support and in direction. Some are mobile robots fitted with handles placed in front of the user ${ }^{5,6}$, but the majority of the systems have the same configuration as the regular four-wheeled walkers. The motorized wheels take place at the back when they are fixed in direction ${ }^{7}$ so as to place the instantaneous center of rotation closer to the user, and indifferently at the back or in front if they are omniwheels ${ }^{8}$.

In all cases the pull and direction of the smart walker are controlled according to a treatment of the efforts of interaction between the handles and the user's hands ${ }^{9,10,11}$. Most of the systems have an obstacle detection function. But few of them can help in Sit-To-Stand ${ }^{12,7,13}$.

These systems are all research prototypes, except PAM-AID which was marketed under GUIDO ${ }^{14}$. Most of them were tested in order to validate their features either on healthy adult subjects, or on the elderly ${ }^{16,17}$. Only PAM-AID was tried on its usability ${ }^{18}$. In that study the PAM-AID was compared to the AMD (Assistive Mobility Device designed by Atlanta VAMC is totally passive and without sensors) and the user's usual walking aid. The test used in the evaluation protocol is a $36,6 \mathrm{~m}$ course, without obstacle, with the three technical devices. The time to complete the tests and a questionnaire were used in the analysis.

This paper is proposing a method to assess the efficiency of smart-walkers. We compare the use of a standard walking frame by four healthy senior volunteers and four elderly patients with some level of motor and cognitive impairment. We are comparing the efficiency of the walkers using the time of task completion and the fit between the human and the walker according to markers on the recorded videos.

\section{MATERIAL AND METHOD}

RobuWALKER (rW) was developed in DOMEO project (AAL-2008-1-159), to be assessed with prospect-users.

\section{Protocol of experiments}

The protocol presented here is part of the objective to estimate the main technological profits expected from the project. The assessment of the use of the technical aid is made from two normalized tests used in geriatric medicine to diagnose frailty and walking difficulties.

These two tests are the 4 Meters Walking Test $^{19}$ (4MWT) and the Timed Get-up and Go Test ${ }^{20}$ (TGG).

The 4 Meters Walking Test measured the time for an elderly person to walk 4 meters, after 2 meters' run up.

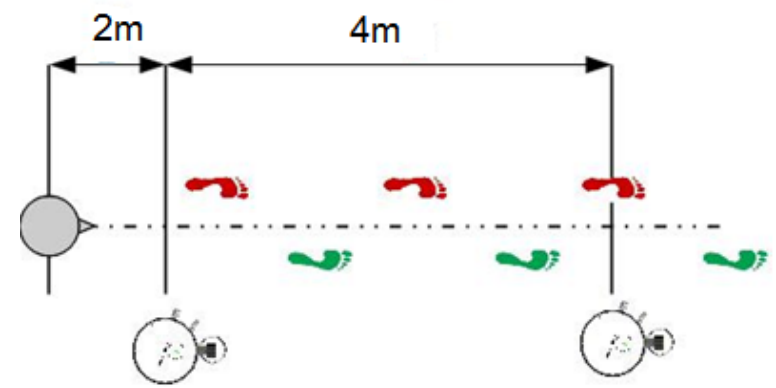

Fig. 1. $4 m$ Walking Test

In our modified TGG, a chair is facing a wall (in our case $3 \mathrm{~m}$ plus the length of the walker). The tester is first telling the volunteer he will have to stand, walk to the wall, turn back and go round the chair and seat down. We chose that version as we thought managing the walking aid around a fixed obstacle could bring extra information. TGG consists in a succession of basic mobility tasks: getting up from a chair, walking, turning round, walking back to the chair, going round the chair, sitting down.

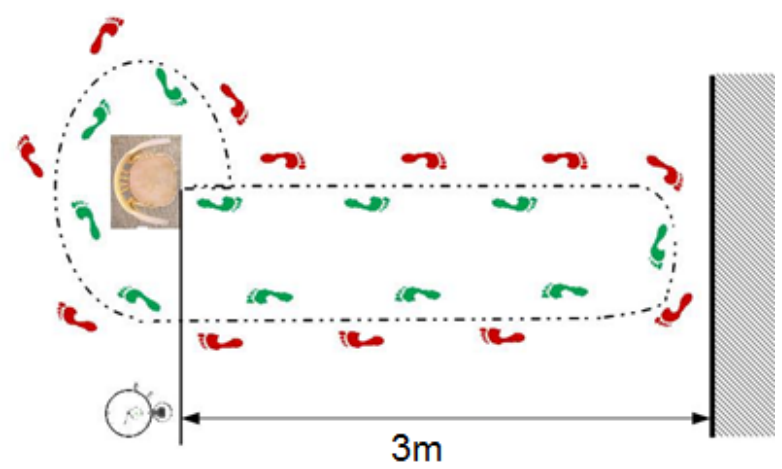

Fig. 2. Modified Timed Get-up and Go Test

\section{Description of the experiment}

Users volunteered after information by a geriatrician from the Toulouse Gérontopôle geriatric medicine department. We chose to propose participation to people, with motor (as assessed by $4 \mathrm{M}$ over 4sec and TGG over 13sec) and cognitive impairment (as assessed by MMSE below 26), and their main natural caregiver as healthy volunteer (for ethical reasons regarding consent of the cognitively impaired person). Participants should not have been using a walking frame prior to the tests. The recruiting was not random. Informed consent was obtained from both healthy and impaired volunteers. In the case of the cognitively impaired, the consent was also given by the main caregiver. No participant was under legal protection. All participants consented in being filmed and allowed films to be used for the purpose of the research.

The protocol would propose first the $4 \mathrm{M}$, then the TGG. If the volunteer failed the $4 \mathrm{M}$, the protocol would stop at that stage. Failed $4 \mathrm{M}$ was considered 
as taking over ten times more time to complete the test with a device then walking the usual way of the volunteer (i.e.: with no aid or a different aid to a walker such as a cane). During each test, the volunteer was first trained to complete the test, then to complete it with first the regular walking frame then the robuWALKER ( $\mathrm{rW}$ ). Training lasted as long as the volunteer required to feel sufficiently confident. Each test was run right after training with a new device (usual way of walking then walking frame and rW).

Each training and test was filmed by a single camera, we had to choose the best point of view and this was left to the cameraperson. Total duration of step and double contact were assessed on a film section where the feet were visible, on a straight line with 3 steps or more at stable speed. This could be on $4 \mathrm{M}$ or a straight portion of TGG.

\section{robuWALKER the smart-walker used}

As to validate that protocol we used rW manufactured by Robosoft ${ }^{21}$. rW is an indoor mobility platform designed for walking and sit-to-stand aid to elderly persons. Regarding mobility this technical aid is composed of 2 driving rear wheels and 2 caster wheels at the front. It has two motorized arms in order to help the elderly to stand from seated. Note that rW verifies the space standards for a wheelchair (Table 1).

\begin{tabular}{|l|l|}
\hline Dimensions & $\mathrm{L} \times 1 \times \mathrm{h}=973 \times 570 \times 560 \mathrm{~mm}$ \\
\hline Ground clearance & $50 \mathrm{~mm}$ \\
\hline Max speed & $0.5 \mathrm{~m} / \mathrm{s}$ \\
\hline
\end{tabular}

Table 1. robuWALKER technical characteristics

$\mathrm{rW}$ is allowing forward and backward motion, turn to the right and left and rising and lowering the handles. 2 buttons are offering the 3 functionalities (Fig.3). The button on the right handle allows:

Walking forward (arrow at the front) or walking backward (arrow at the back),

Turning right (right arrow) or turning left (left arrow), The button on the left handle allows rising the two handles at the same pace (up arrow) or lowering the two handles at the same pace (down arrow).

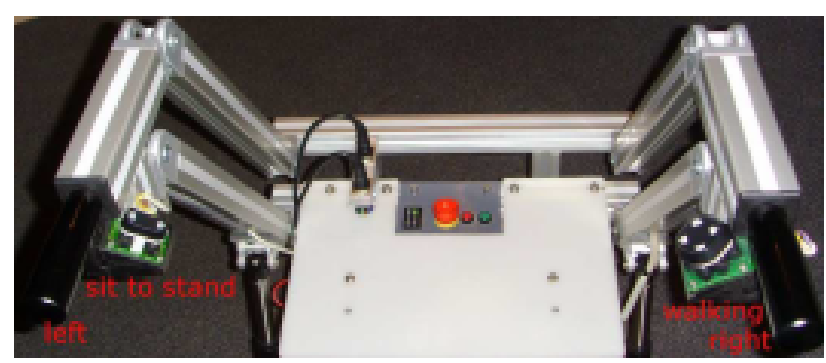

Fig. 3. robuWALKER buttons to control functionalities

\section{RESULTS}

\section{Population}

Nine persons were included in the protocol: four healthy volunteers aged 71 to 86 ; five impaired volunteers aged 83 to 96 . One healthy volunteer proved to have walking impairment (YCA) with $4 \mathrm{M}$ at $5.7 \mathrm{sec}$ and TGG at $19 \mathrm{sec}$. As she had no cognitive impairment she had to be excluded from analysis. One impaired volunteer (MCO) had improved with MMSE $=26,4 \mathrm{M}$ at 3.28 and $\mathrm{TG}$ at 12.24 and was thus requalified as healthy volunteer. Their characteristics are displayed in Table 2.

\begin{tabular}{|l|l|l|l|l|l|c|}
\hline User & Age & Gender & Size & Weight & $\begin{array}{c}\text { With } \\
\text { walking } \\
\text { trouble }\end{array}$ & MMS \\
\hline CAR & 75 & Female & 1.72 & 49 & no & irrelevant \\
\hline HRU & 71 & Female & 1.58 & 57 & no & irrelevant \\
\hline YCA & 86 & Female & 1.63 & 62 & no & irrelevant \\
\hline YCO & 77 & Female & 1.61 & 55,5 & yes & irrelevant \\
\hline MCO & 80 & Male & 1.75 & 73 & no & 26 \\
\hline FSE & 96 & Male & 1.70 & 75 & yes & 19 \\
\hline GCA & 83 & Male & 1.72 & 80 & yes & 19 \\
\hline MGO & 86 & Female & 1.48 & 55 & yes & 17 \\
\hline LSE & 93 & Female & 1.56 & 55 & yes & 25 \\
\hline
\end{tabular}

Table 2. The subjects

\section{Time of completion}

In table 3 we compare the time to complete each test for each of the eight volunteers. Six of them completed the two tests under the three different conditions.

One of them (LSE) could not succeed in the $4 \mathrm{M}$ test with $\mathrm{rW}$ and then was not proposed the TGG. One of them (MCO) could not succeed at TGG with rW.

\begin{tabular}{|l|l|l|l|l|l|l|}
\hline & \multicolumn{3}{|c|}{ 4M } & \multicolumn{3}{c|}{ TGG } \\
\hline Users & usual & frame & rW & usual & frame & W \\
\hline CAR & 2.92 & 2.70 & 17.19 & 11.74 & 23,98 & 90,76 \\
\hline HRU & 2.65 & 2.52 & 15.12 & 9.90 & 13.68 & 58.59 \\
\hline YCA & 5.7 & 5.4 & 15 & 19 & 30.7 & 58 \\
\hline MCO & 3.28 & 2.56 & 26.64 & 12.24 & 21.91 & 132.48 \\
\hline FSE & 5.31 & 6.70 & 14.85 & 27.99 & 31.68 & 82.57 \\
\hline GCA & 8.68 & 8.77 & 10.66 & 34.24 & 52.69 & 99.54 \\
\hline MGO & 4.59 & 3.87 & 8.73 & 24.03 & 30.15 & 54.09 \\
\hline LSE & 8.86 & 12.73 & 119.65 & X & X & X \\
\hline
\end{tabular}

Table 3. Time in seconds to perform tasks

\section{Analysis of videos}

It was possible for each user to asses the expected values. Tables 4 shows the durations of steps with the regular walking frame (D) and rW, the ration between those, the ratio of one double contact to the total duration of step with $D$ and $r W$, then the difference of those ratios. In elderly healthy users, the duration of step with $\mathrm{rW}$ increases 1.8 to 3.4 times, double contact 4 to 9 times, the median increase of double contact ratio is .15 . In impaired users there is a lesser or no increase in step 1 to 1.5 , a lesser increase in double contact 1.3 to 2 , the double contact ratio just increasing 05 . 


\begin{tabular}{|c|c|c|c|c|c|c|}
\hline Users & $\begin{array}{c}\text { step } \\
\text { duration } \\
\text { D }\end{array}$ & $\begin{array}{c}\text { step } \\
\text { duration } \\
\text { rW }\end{array}$ & ratio & $\begin{array}{c}\text { double } \\
\text { contact } D\end{array}$ & $\begin{array}{c}\text { double } \\
\text { contact rW }\end{array}$ & $\begin{array}{c}\text { ratio } \\
\text { difference }\end{array}$ \\
\hline CAR & 1,04 & 3,52 & 3,38 & 0,11 & 0,96 & 0,17 \\
\hline HRU & 0,96 & 1,84 & 1,92 & 0,08 & 0,40 & 0,13 \\
\hline YCO & 1,28 & 2,60 & 2,03 & 0,16 & 0,40 & 0,03 \\
\hline MCO & 1,04 & 1,92 & 1,85 & 0,16 & 0,64 & 0,18 \\
\hline FSE & 1,52 & 2,24 & 1,47 & 0,24 & 0,48 & 0,06 \\
\hline GCA & 1,60 & 2,09 & 1,31 & 0,32 & 0,48 & 0,03 \\
\hline LES & 1,52 & 1,52 & 1,00 & 0,24 & 0,40 & 0,11 \\
\hline MGO & 1,20 & 1,36 & 1,13 & 0,24 & 0,32 & 0,04 \\
\hline
\end{tabular}

Table 4. Characteristics of steps with either aids

\section{Discussion}

Due to the small amount of time of availability of the rW we chose to select people with no previous experience of walkers to avoid a training bias against the tested device. Completion times are showing that the tested walker is less efficient than a regular walking frame, but the smaller effect on the profile of steps as compared to that on healthy users could be a clue of a better adaptation to those in need. Healthy users could be pushing the rW due to it's nominal speed being to slow for them. Two patients failed in using the device, this might be due, if we consider that speed and ergonomics are fitting (from the effect on step parameters) to a human robot interaction (HRI) problem; we could see that from the clinical analysis of posture and use on the videos. Quantified data could not asses getting up. In further tests we will increase the number of cameras to have different points of view to avoid dead angles and allow for posture analysis (tilt, distance of body to handles, special issue of getting up) and have a special focus on HRI (that would not be allowed by optokinetics).

\section{References}

1. Glover, J., Holstius, D., Manojlovich, M., Montgomery, K., Powers, A., Wu, J., Kiesler, S., Mattews, J., Thrun, S., A Robotically-Augmented Walker for Older Adults, CMU-CS-03-170, Carnegie Mellon University, August 2003.

2. Hirata, Y., Muraki, A., Kosuge, K., Motion Control of Intelligent Passive-type Walker for Fallprevention Function Based on Estimation of User State, in Proceedings of the 2006 IEEE International Conference on Robotics and Automation, pp. 3498-3503, 2006.

3. Lacey, G., Dawson-Howe, K., The application of robotics to a mobility aid for the elderly blind, in Robotic Automation Systems, 23(4), pp. 245-252, 1998. 4. C. Huang, G. Wasson, M. Alwan, P. Sheth, A. Ledoux; Shared Navigational Control and User Intent Detection in an Intelligent Walker, In Proceedings of AAAl Fall 2005 Symposium (EMBC), 2005.

5. Graf, B., Hans, M., Schraft, R., Care-O-bot II Development of a next generation robotic home assistant; Autonomous Robots, 16(2):193-205, 2004.
6. Morris, R. Donamukkala, A. Kapuria, A. Steinfield, J. Matthews, J. Dunbar-Jacobs, S. Thrun; A Robotic walker that provides guidance, In Proceedings of IEEE International Conference on Robotics and Automation (ICRA), Taipei, Taiwan, 2003.

7. Chugo, D., Matsuoka, W., Jia, S., Takase, K., A Robotic walker with Standing Assistance, Proceedings of the 2008 IEEE International Conference on Information and Automation, pp. 452-457, 2008.

8. Yu, H., Spenko, M., Dubowsky, S., Omni-directional mobility using active split offset castors, ASME Journal of Mechanical Design, 126(5), pp. 822-829, Sept. 2004.

9. Egawa, S., Takeuchi, I., Koseki, A., Ishii, T., Electrically Assisted Walker with Supporterembedded Force-sensing Device, In Advances in Rehabilitation Robotics: Human-friendly Technologies on Movement Assistance and Restoration for People with Disabilities, Vol. 306, pp.313322, 2004.

10. Chugo, D., Matsuoka, W., Sogmin, J., Takase, K., A Rehabilitation Walker with standing-Assistance Device, Journal of Robotics and Mechatronics, Vol. 19(6), pp. 604-611, 2007.

11. Saint-Bauzel, L., Pasqui, V., Monteil, I., A reactive robotized interface for lower limb rehabilitation: clinical results. IEEE Transaction on Robotics, Special Issue on Rehabilitation Robotics. Vol 25(3), pp. 583592, 2009.

12. Nemoto, Y., et al., Power Assist Control for Walking Support System, in Proceedings of the 9th International Conference on Advanced Robotics, pp. 1518, 1999.

13. Médéric, P., Pasqui, V., Plumet, F., Bidaud, Ph., Sit to Stand Transfer Assisting by an Intelligent Walking-Aid. CLAWAR04: 7th International Conference on Climbing and Walking Robots. Espagne, 2004.

14. Retrieved from: www.haptica.com

15. Alwan, M., Rajendran, P.J., Ledoux, A., Huang, C., Wasson, G., Sheth, P., Stability margin Monitoring in Steering-Controlled Intelligent Walkers for the Elderly, In Proceedings of AAAI Symposium, 2005.

16. Yu, H., Spenko, M., Dubowsky, S., An adaptive shared control system for an intelligent mobility aid for the elderly, Autonomous Robots, Vol. 15, pp. 53-66, 2003.

17. Graf, B., An adaptive Guidance system for Robotic Walking aids, Journal of Computing and Information Technology, Vol. 1, pp. 109-120, 2009.

18. Rentschler, Simpson, R., Cooper, R., Boninger, M., Clinical evaluation of Guido robotic walker, Journal of Rehabilitation Research \&Development, Vol. 45, pp. 1281-1294, 2008.

19. Rolland, Y. M., Cesari, M., Miller, M., Penninx, B., Atkinson, H., Pahor M., Reliability of the 400-M Usual-PaceWalk Test as an Assessment of Mobility Limitation Older Adults, J Am Geriatr Soc 52:972-976, 2004.

20. Podsiadlo, D., Richardson, S., The timed 'Up and Go' Test: a Test of Basic Functional Mobility for 
Frail Elderly Persons. Journal of American Geriatric Society. Vol. 39, pp. 142-148, 1991.

21. Retrieved from: http://www.robosoft.com

22. Fried, L.P., Tangen, C.M., Walston, J., et al., Frailty in older adults: evidence for a phenotype. J. Gerontol A Biol Sci Med Sci Vol. 56(3), pp. M146$56,2001$. 